\title{
Narrow-aperture electron beam in the forevacuum pressure range as a tool for dimensional processing of silica glass
}

\author{
Aleksey Zenin ${ }^{1, *}$, Aleksandr Klimov $^{1}$, and Ilya Bakeev ${ }^{1}$ \\ ${ }^{1}$ Tomsk State University of Control Systems and Radioelectronics, 634040 Tomsk, Russia
}

\begin{abstract}
The paper gives overview of the results of using narrowaperture electron beam generated by a forevacuum plasma electron source as a tool for treatment of electrically non-conductive materials, such as technical glass. Effectiveness of electron beam treatment of nonconductive materials is caused by almost complete neutralization of target charge on the treated surface. Charge neutralization occurs due to ion flow from beam plasma generated while transporting the beam through forevacuum pressure area. The study demonstrates the possibility to control depth and form of milling by changing modes of electron beam treatment. Combined use of electron beam and automated system for beam deflection and sweep makes possible to perform dimensional processing, particularly cutting and milling with complex trajectory. Milling rates were experimentally found depending on treatment time and mode. The suggested method of silica glass treatment represents an alternative for traditional treatment methods.
\end{abstract}

\section{Introduction}

Due to the unique properties, such as high electric strength, low coefficient of thermal expansion, and high chemical resistance, silica glass is widely used in numerous industries [1]. The main silica glass treatment methods consist in either mechanical removal of foreign materials and polish or thermal treatment. Mechanical treatment often causes formation of cracks and microcracks in treated material, which considerably decreases strength parameters of products [2]. Thermal treatment (by torch flame) does not allow producing holes with complex shape and clearly defined dimensions and may add carbon impurities in the material composition, which has negative effect on its optical properties [3].

The technology of laser controlled thermal splitting can be applied for cutting thin glass products with maximum thickness of $3 \mathrm{~mm}$ [4]. Whereas electron beam treatment can be effectively used for performing operations with thick-wall silica glass products and products with complex shape, as well as for removing shortcomings of the existing treatment methods [5-8]. However, electron beams which normally function in high

*Corresponding author: Zenin1988@gmail.com 
vacuum (up to $10^{-1} \mathrm{~Pa}$ ) are difficult to use for dielectric treatment due to accumulation of negative surface charge in the treated product [8].

Advance of plasma electron sources able to function in forevacuum pressure areas between 10 and $100 \mathrm{~Pa}$ [8] considerably extend opportunities for electron beam treatment of electric non-conductive materials and for their surface modification. Hence, due to presence of dense plasma in the beam transport area such electron sources are capable of performing treatment of both conductive materials and products and dielectrics without any means of surface charge compensation. Effectiveness of electron beam treatment of dielectrics using such sources is showed using the example of welding and vaporization of ultra-high temperature ceramics, sintering of ceramics, and polymer surface modification $[9,10]$.

Previous studies of the research group on obtaining narrow-aperture electron beams in forevacuum pressure area [11] open up new opportunities of electron beam technology, such as precision milling and dielectric cutting and sizing. This research is focused on studying the interaction between silica glass and electron beam generated by forevacuum plasma source to demonstrate opportunities of using such beam as an alternative sizing tool.

\section{Experimental method}

The experiment was performed using a vacuum setup (Fig. 1) with an installed forevacuum electron plasma source.

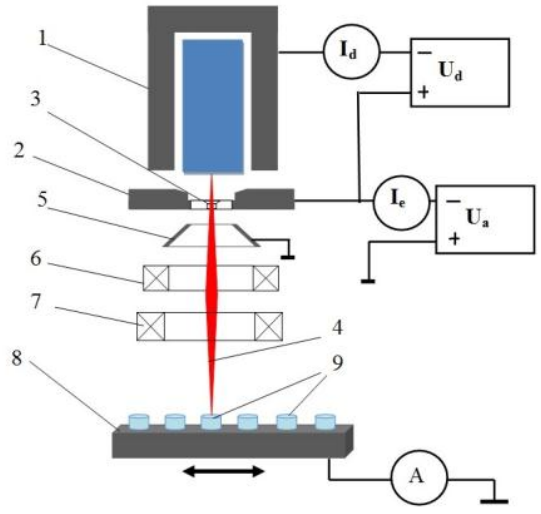

Fig. 1. Experimental unit for measuring shape and depth of milling: 1 - hollow cathode, 2 - anode, 3 - perforated electrode, 4 - electron beam, 5 - accelerating electrode, 6 - focusing system, 7 deflection system, 8 - carriage, 9 - treated specimens.

Emission plasma in the source was generated by glow discharge with hollow cathode 1 . Electron beam was formed by extracting electrons from plasma boundary through the hole in perforated electrode 3 installed in anode 2 . Accelerated electron beam 4 was focused by magnetic lens 5 and was directed to the treated specimen 9 using magnetic beam deflection system 6 . The electrode system and structural peculiarities of electron source, as well as its parameters and characteristics are described in more detail in the works [8-10]. Silica glass rods having $10 \mathrm{~mm}$ in diameter and $30 \mathrm{~mm}$ in length were used for investigation of hole form and milling depth. Use of movable carriage allowed performing a series of experiments in a single process cycle [11]. In order to measure the hole form and milling depth, the time of specimen exposure to electron beam was set in the range between 10 and 60 seconds, accelerating voltage was constant and was set in the range between 10 and 19 $\mathrm{kV}$. The beam current was constant during the experiment and was equal to $10 \mathrm{~mA}$. The 
beam had the diameter of $1 \mathrm{~mm}$ and was controlled by the parameters of focusing system. Helium was used as working gas with the pressure of $30 \mathrm{~Pa}$.

\section{Results and discussion}

Experimental studies on electron beam treatment of silica glass showed that intense material vaporization occurs at accelerating voltage of $10 \mathrm{kV}$ and a molten pool is formed in the treated area. Due to this effect, deepening of the region of electron beam absorption causes formation of knife-like cavitation, i.e. a capillary the depth of which is several times greater that the orifice diameter. Figure 2 presents various characteristic forms of capillaries formed at different accelerating voltages.
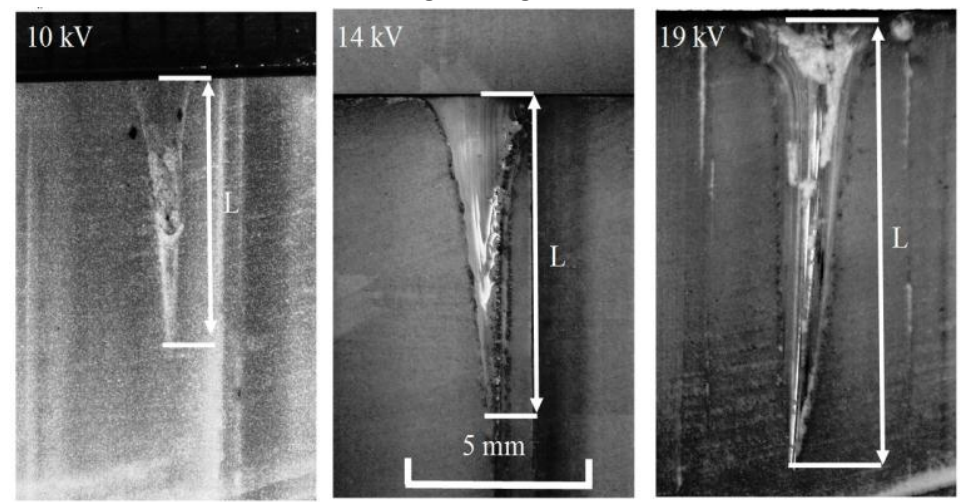

Fig. 2. Cutoffs of obtained cavitations, treatment time $50 \mathrm{~s}$.

The observed intense material evaporation and formation of knife-like cavitation is an effective means of silica glass cutting. Milling depth is precisely controlled by the electron beam parameters and treatment time. This way, the dependence of milling depth on accelerating voltage is almost linear (Fig. 3). However, the dependence of treatment time on penetration depth has a more complex nature (Fig. 4). With treatment time less than 30 seconds the milling depth grows almost independently of accelerating voltage. When increasing treatment time, the depth difference grows for various accelerating voltage values.

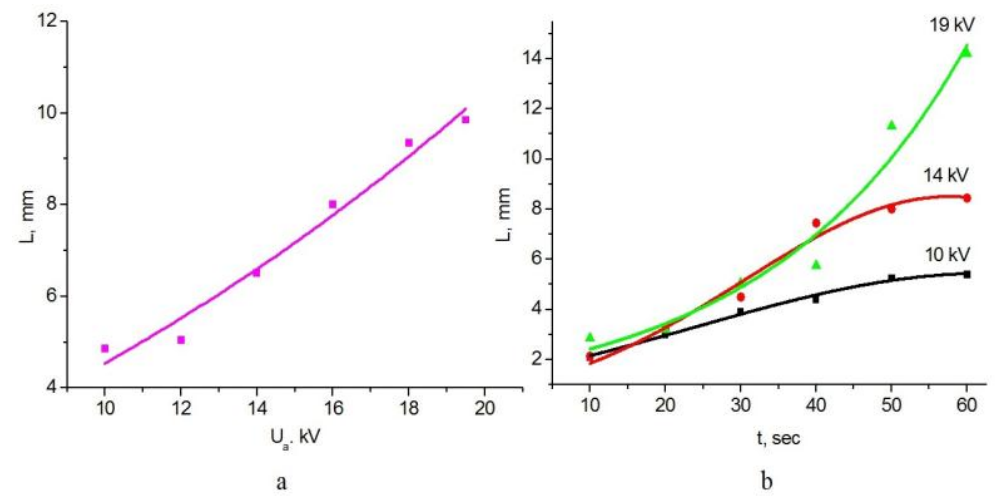

Fig. 3. Dependence of milling depth on electron beam treatment parameters: a) on accelerating voltage with treatment time of 40 seconds, b) on treatment time for various accelerating voltage values. 
Figure 4 demonstrates dependences of removed mass on treatment time for various accelerated voltage values. These dependences are obviously similar to the above dependences of penetration depth on treatment time. However, certain differences can be found, such as ablative mass with constant milling depth for accelerating voltage of 10 and $14 \mathrm{kV}$, which can be related to minor change of electron beam diameter.

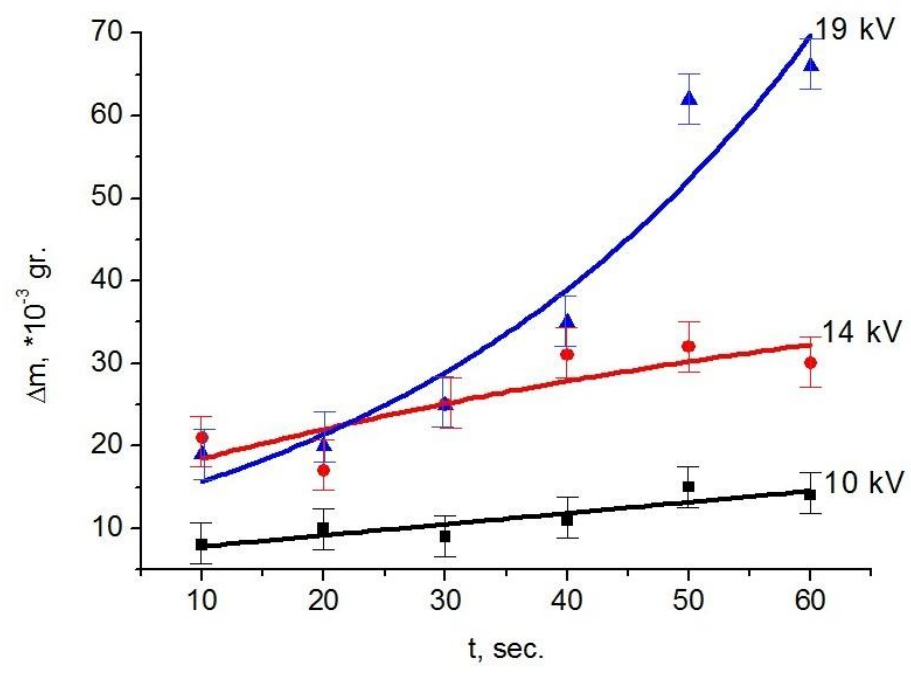

Fig. 4. Dependence of ablative mass on treatment time and electron beam parameters.

The given dependences demonstrate that, as one could expect, increase of accelerating voltage causes proportionate growth of milling depth (Fig. 3). In this way, increase of accelerating voltage from 10 to $19 \mathrm{kV}$ almost doubles the milling depth. Change of duration of electron beam treatment of a silica glass specimen also results in increasing the milling depth.

The use of automated system for electromagnetic beam deflection allows moving the beam with complex trajectory at a given rate, which enables to cut long objects with intricate shape. Below, Figure 5 presents an example of using finely focused electron beam for silica glass sizing. Silica plate $300 \times 50 \mathrm{~mm}$ in size and $4 \mathrm{~mm}$ thick was used as test material for milling. Accelerated voltage was $18 \mathrm{kV}$, full milling time was about 4 minutes, the number of passes the electron beam performs over a specimen was 15 .

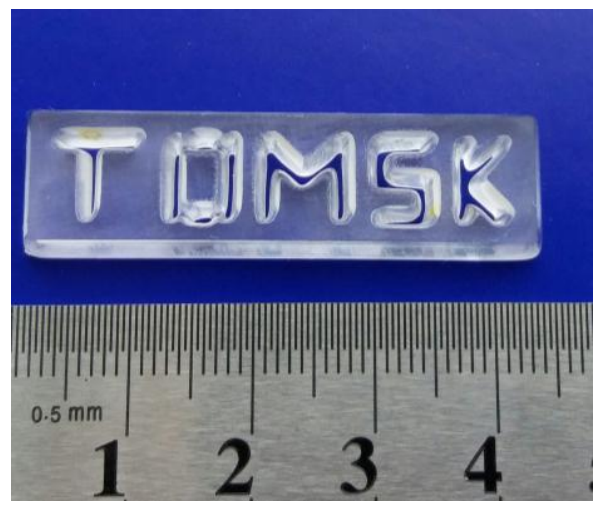

Fig. 5. Example of electron beam milling of a silica plate. 
It should be noted that no cracks are observed on the edges of the formed cavitation. The developed treatment method allows avoiding crack formation in treated products, as well as treating objects with intricate shape. Automated beam positioning system allows building complex trajectories while treating objects.

\section{Conclusion}

The performed research demonstrates that a knife-like cavitation is formed while treating silica glass specimens with electron beam. The milling depth (provided the transverse beam size is constant) can be controlled by varying accelerating voltage and treatment time. Increase of both accelerating voltage and treatment time results in growth of depth.

Removed mass rate while milling may vary between 10 and $70 \mathrm{mg}$ per minute depending on electron beam treatment parameters. The research demonstrates the possibility of silica glass dimensional cutting and milling with complex trajectory and depth control by means of beam positioning and sweep with the help of automated system for electromagnetic beam deflection. The suggested electron beam treatment method for silica glass represents an alternative for traditional treatment methods. Further extension of the research shall be reduction of electron beam diameter for reducing geometrical parameters of holes, as well as investigation of peculiarities of electron-beam heating of glass with the purpose of welding.

The work has been conducted with the financial support of Russian Foundation for Basic Research, project No.16-38-60032 mol_a_dc.

\section{References}

1. N.P. Bansal, R.H. Doremus, Handbook of glass properties (Elsevier, New York, 2013)

2. P.V. Volkov, A.N. Bryzgalov, D.E Zhivulin, Scientific achievements in biology, chemistry, physics, Proceedings of VI International Scientific Conference (Novosibirsk, April 4, 2012) (in Russian)

3. A.Y. Legoshin, L.A. Manuylov, Stekloduvnoe delo [Glassblowing] (Vysshaya Shkola Publ., Moscow, 1976) (in Russian)

4. V. Kondratenko, P.S. Tchernykh, Phys. Stat. Sol. 7, 2232 (2007)

5. S. Kornilov, N. Rempe, A. Beniyash, N. Murray, Journal Of Physics: conference series 552(1), 012004 (2014)

6. M.St. Weglowski, S. Błacha, A. Phillips, Vacuum 130, 72-92 (2016)

7. T.K. Saha, M. Mascarenhas, E. Kandaswamy, The International Symposia on Discharge and Electrical Insulation in Vacuum (ISDEIV) (Mumbai, September 28October 3, 2014)

8. A. Zenin, A. Klimov, V. Burdovitsin, E. Oks, Technical Physics Letters 39(10), 454456 (2013)

9. V.A. Burdovitsin, E.M. Oks, Laser and particle beams 26, 04, 619-635 (2008)

10. A.A. Zenin, I.Y. Bakeev, Y.A. Burachevskii, A.S. Klimov, E.M. Oks, Technical Physics Letters 42(7), 712-714 (2016)

11. S. Schiller, U. Heisig, S. Panzer, Electronenstrahltechnologie (VEB Verlag Technik, Dresden, 1976) 